\title{
Nonlinear instability of inhomogeneous steady states solutions to the HMF Model
}

\author{
M. Lemou ${ }^{\dagger}$, A. M. Luz ${ }^{\star}$, and F. Méhats ${ }^{\ddagger}$ \\ ${ }^{\dagger}$ Univ Rennes, CNRS, IRMAR, mohammed.lemou@univ-rennes1.fr \\ ${ }^{\star}$ IME, Universidade Federal Fluminense, analuz@id.uff.br \\ ‡Univ Rennes, IRMAR, florian.mehats@univ-rennes1.fr
}

April 2, 2019

\begin{abstract}
In this work we prove the nonlinear instability of inhomogeneous steady states solutions to the Hamiltonian Mean Field (HMF) model. We first study the linear instability of this model under a simple criterion by adapting the techniques developed in [19]. In a second part, we extend to the inhomogeneous case some techniques developed in [14, 17, 18, and prove a nonlinear instability result under the same criterion.
\end{abstract}

\section{Introduction}

In this paper, we are interested in the nonlinear instability of inhomogeneous steady states of the Hamiltonian Mean Field (HMF) system. The HMF system is a kinetic model describing particles moving on a unit circle interacting via an infinite range attractive cosine potential. This 1D model holds many qualitative properties of more realistic long-range interacting systems as the Vlasov-Poisson model. The HMF model has been the subject of many works in the physical community, for the study of non equilibrium phase transitions [11, 26, 2, 24, of travelling clusters [6, 27] or of relaxation processes [28, 3, 12]. The long-time validity of the N-particle approximation for the HMF model has been investigated in [8, 9] and the Landau-damping phenomenon near a spatially homogeneous state has been studied recently in [13]. The formal linear stability of inhomogeneous steady states has been studied in [10, 23, 7]. In particular, a simple criterion of linear stability has been derived in [23]. In [22], the authors of the present paper have proved that, under the same criterion $\kappa_{0}<1$ (see below for a precise formulation), the inhomogeneous steady states of HMF that are nonincreasing functions of the microscopic energy are nonlinearly stable. The aim of the present paper is to show, in a certain sense, that this criterion is sharp: we show that if $\kappa_{0}>1$, the HMF model can develop instabilities, from both the linear and the nonlinear points of view. 
In [15], Guo and Lin have derived a sufficient criterion for linear instability to 3D Vlasov-Poisson by extending an approach developped in [19] for BGK waves. Let us also mention that both works have adapted some techniques presented in [16] to prove the nonlinear instability of the 3D Vlasov-Poisson system. In the first part of this article we adapt these techniques and prove the linear instability of nonhomogeneous steady states to the HMF system. In [17, a nonlinear instability result for 1D Vlasov-Poisson equation was obtained for an initial data close to stationary homogeneous profiles that satisfy a Penrose instability criterion by using an approach developed in [14]. In [18, starting with the N- particles version of the HMF model, a nonlinear instability result is obtained for the corresponding Vlasov approximation by also considering a Penrose instability condition for stationary homogeneous profiles. In the second part of this article our aim is to prove the nonlinear instability of non-homogeneous steady states of HMF by adapting the techniques developed in [14, 17, 18].

In the HMF model, the distribution function of particles $f(t, \theta, v)$ solves the initialvalued problem

$$
\begin{aligned}
& \partial_{t} f+v \partial_{\theta} f-\partial_{\theta} \phi_{f} \partial_{v} f=0, \quad(t, \theta, v) \in \mathbb{R}_{+} \times \mathbb{T} \times \mathbb{R}, \\
& f(0, \theta, v)=f_{\text {init }}(\theta, v) \geqslant 0,
\end{aligned}
$$

where $\mathbb{T}$ is the flat torus $[0,2 \pi]$ and where the self-consistent potential $\phi_{f}$ associated with a distribution function $f$ is defined by

$$
\phi_{f}(\theta)=-\int_{0}^{2 \pi} \rho_{f}\left(\theta^{\prime}\right) \cos \left(\theta-\theta^{\prime}\right) d \theta^{\prime}, \quad \rho_{f}(\theta)=\int_{\mathbb{R}} f(\theta, v) d v .
$$

Introducing the so-called magnetization vector defined by

$$
M_{f}=\int_{0}^{2 \pi} \rho_{f}(\theta) u(\theta) d \theta, \quad \text { with } \quad u(\theta)=(\cos \theta, \sin \theta)^{T}
$$

we have

$$
\phi_{f}(\theta)=-M_{f} \cdot u(\theta) .
$$

In this work will consider steady states of (1.1) of the form

$$
f_{0}(\theta, v)=F\left(e_{0}(\theta, v)\right),
$$

where $\mathrm{F}$ is a given nonnegative function and where the microscopic energy $e_{0}(\theta, v)$ is given by

$$
e_{0}(\theta, v)=\frac{v^{2}}{2}+\phi_{0}(\theta) \quad \text { with } \phi_{0}=\phi_{f_{0}} .
$$

Without loss of generality, we assume that $\phi_{0}(\theta)=-m_{0} \cos \theta$ with $m_{0}>0$. Here $m_{0}$ is the magnetization of the stationary state $f_{0}$ defined by $m_{0}=\int \rho_{f_{0}} \cos \theta d \theta$.

It is shown in [22] that (essentially) if $F$ is decreasing then $f_{0}$ is nonlinearly stable by the HMF flow (1.1) provided that the criterion $\kappa_{0}<1$ is satisfied, where $\kappa_{0}$ is given 
by

$\kappa_{0}=-\int_{0}^{2 \pi} \int_{-\infty}^{+\infty} F^{\prime}\left(e_{0}(\theta, v)\right)\left(\frac{\int_{\mathcal{D}_{e_{0}(\theta, v)}}\left(\cos \theta-\cos \theta^{\prime}\right)\left(e_{0}(\theta, v)+m_{0} \cos \theta^{\prime}\right)^{-1 / 2} d \theta^{\prime}}{\int_{\mathcal{D}_{e_{0}(\theta, v)}}\left(e_{0}(\theta, v)+m_{0} \cos \theta^{\prime}\right)^{-1 / 2} d \theta^{\prime}} d \theta d v\right.$,

with

$$
\mathcal{D}_{e}=\left\{\theta^{\prime} \in \mathbb{T}: m_{0} \cos \theta^{\prime}>-e\right\} .
$$

In this paper, we explore situations where this criterion is not satisfied, i.e when $\kappa_{0}>1$. Let us now state our two main results. The first one concerns the linearized HMF equation given by

$$
\partial_{t} f=L f
$$

where

$$
L f:=-v \partial_{\theta} f+\partial_{\theta} \phi_{0} \partial_{v} f+\partial_{\theta} \phi_{f} \partial_{v} f_{0} .
$$

Theorem 1.1 (Linear instability). Let $f_{0} \in L^{1}(\mathbb{T}, \mathbb{R})$ be a stationary solution of (1.1) of the form (1.5), where $F$ is a nonnegative $\mathcal{C}^{1}$ function on $\mathbb{R}$ such that $F^{\prime}\left(e_{0}(\theta, v)\right)$ belongs to $L^{1}(\mathbb{T}, \mathbb{R})$. Assume that $\kappa_{0}>1$, where $\kappa_{0}$ is given by (1.7). Then there exists $\lambda>0$ and a non-zero $f \in L^{1}(\mathbb{T} \times \mathbb{R})$ such that $e^{\lambda t} f$ is a nontrivial growing mode weak solution to the linearized HMF equation (1.8).

Our second result is the following nonlinear instability theorem.

Theorem 1.2 (Nonlinear instability). Let $f_{0}$ be a stationary solution of (1.1) of the form (1.5), where $F$ is a $\mathcal{C}^{\infty}$ function on $\mathbb{R}$, such that $F(e)>0$ for $e<e_{*}, F(e)=0$ for $e \geqslant e_{*}$, with $e_{*}<m_{0}$ and $\left|F^{\prime}(e)\right| \leqslant C\left|e_{*}-e\right|^{-\alpha} F(e)$ in the neighborhood of $e_{*}$, for some $\alpha \geqslant 1$. Assume that $\kappa_{0}>1$, where $\kappa_{0}$ is given by (1.7). Then $f_{0}$ is nonlinearly unstable in $L^{1}(\mathbb{T} \times \mathbb{R})$, namely, there exists $\delta_{0}>0$ such that for any $\delta>0$ there exists a nonnegative solution $f(t)$ of (1.1) satisfying $\left\|f(0)-f_{0}\right\|_{L^{1}} \leqslant \delta$ and

$$
\left\|f\left(t_{\delta}\right)-f_{0}\right\|_{L^{1}} \geqslant \delta_{0}
$$

with $t_{\delta}=O(|\log \delta|)$ as $\delta \rightarrow 0$.

Remark 1.3. Note that in these two theorems we do not assume that the profile $F$ is a decreasing function. Besides, the set of steady states satisfying the assumptions of these theorems is not empty, as proved in the Appendix (see Lemma A.1). Note also that the instability of Theorem 1.2 is not due to the usual orbital instability. Indeed the functional space of the pertubation can be restricted to the space of even functions in $(\theta, v)$.

The outline of the paper is as follows: Sections 2 and 3 are respectively devoted to the proofs of Theorem 1.1 and Theorem 1.2 . 


\section{A linear instability result: proof of Theorem 1.1}

The aim of this section is to prove Theorem 1.1. This proof will be done following the framework used by Lin for the study of periodic BGK waves in [19], which was generalized to the analysis of instabilities for the 3D Vlasov-Poisson system by Guo and Lin in [15]. We divide this proof into the three Lemmas 2.1, 2.2 and 2.3, respectively proved in Subsections 2.1, Subsection 2.2 and Subsection 2.3.

A growing mode of $(1.8)$ is a solution of the form $e^{\lambda t} f$, where $f \in L^{1}(\mathbb{T}, \mathbb{R})$ is an unstable eigenfunction of $L$, i.e. a nonzero function satisfying $L f=\lambda f$ in the sense of distributions, with $\lambda \in \mathbb{R}_{+}^{*}$ and with $L$ defined by (1.9). Note that the equation $L f-\lambda f=0$ is invariant by translation: if $f(\theta, v)$ is an eigenfunction, then for all $\theta_{0}$, $f\left(\theta+\theta_{0}, v\right)$ is also an eigenfunction. Since for all $f \in L^{1}$ we can find a $\theta_{0} \in \mathbb{T}$ such that $\int \rho_{f}\left(\theta+\theta_{0}\right) \sin \theta d \theta=0$, we can assume that our eigenfunction of $L$ always satisfy $\int \rho_{f} \sin \theta d \theta=0$, i.e. $\phi_{f}=-m \cos \theta$ with $m=\int \rho_{f} \cos \theta d \theta$.

Let us first define $(\Theta(s, \theta, v), V(s, \theta, v))$ as the solution of the characteristics problem

$$
\left\{\begin{array}{l}
\frac{d \Theta(s, \theta, v)}{d s}=V(s, \theta, v) \\
\frac{d V(s, \theta, v)}{d s}=-\partial_{\theta} \phi_{0}(\Theta(s, \theta, v))
\end{array}\right.
$$

with initial data $\Theta(0, \theta, v)=\theta, V(0, \theta, v)=v$. When there is no ambiguity, we denote simply $\Theta(s)=\Theta(s, \theta, v)$ and $V(s)=V(s, \theta, v)$. Since $\phi_{0}(\theta)=-m_{0} \cos \theta$, the solution $(\Theta, V)$ is globally defined and belongs to $\mathcal{C}^{\infty}(\mathbb{R} \times \mathbb{T} \times \mathbb{R})$. Note that the energy $e_{0}(\Theta(s), V(s))=\frac{V(s)^{2}}{2}+\phi_{0}(\Theta(s))$ does not depend on $s$.

We shall reduce the existence of a growing mode of (1.8) to the existence of a zero of the following function, defined for all $\lambda \in \mathbb{R}_{+}^{*}$ :

$$
\begin{aligned}
G(\lambda)=1 & +\int_{0}^{2 \pi} \int_{\mathbb{R}} F^{\prime}\left(\frac{v^{2}}{2}-m_{0} \cos \theta\right) \cos ^{2} \theta d \theta d v \\
& -\int_{0}^{2 \pi} \int_{\mathbb{R}} F^{\prime}\left(\frac{v^{2}}{2}-m_{0} \cos \theta\right)\left(\int_{-\infty}^{0} \lambda e^{\lambda s} \cos \Theta(s, \theta, v) d s\right) \cos \theta d \theta d v .
\end{aligned}
$$

Lemma 2.1. Let $f_{0} \in L^{1}(\mathbb{T}, \mathbb{R})$ be a stationary solution of (1.1) of the form (1.5), where $F$ is a $\mathcal{C}^{1}$ function on $\mathbb{R}$ such that $F^{\prime}\left(e_{0}(\theta, v)\right)$ belongs to $L^{1}(\mathbb{T}, \mathbb{R})$. Then the function $G$ defined by (2.2) is well-defined and continuous on $\mathbb{R}_{+}^{*}$. Moreover, there exists a growing mode $e^{\lambda t} f$ solution to (1.8) associated with the eigenvalue $\lambda>0$ if and only if $G(\lambda)=0$. An unstable eigenfunction $f$ of $L$ is defined by

$$
f(\theta, v)=-F^{\prime}\left(e_{0}(\theta, v)\right) \cos \theta+F^{\prime}\left(e_{0}(\theta, v)\right) \int_{-\infty}^{0} \lambda e^{\lambda s} \cos (\Theta(s, \theta, v)) d s .
$$

Lemma 2.2. Under the assumptions of Lemma 2.1, the function $G$ defined by (2.2) satisfies

$$
\lim _{\lambda \rightarrow 0^{+}} G(\lambda)=1-\kappa_{0}
$$


where $\kappa_{0}$ is defined by (1.7).

Lemma 2.3. Under the assumptions of Lemma 2.1, the function $G$ defined by (2.2) satisfies

$$
\lim _{\lambda \rightarrow+\infty} G(\lambda)=1 .
$$

Proof of Theorem 1.1. From these three lemmas, it is clear that if $\kappa_{0}>1$, we have $\lim _{\lambda \rightarrow 0^{+}} G(\lambda)<0$ and $\lim _{\lambda \rightarrow+\infty} G(\lambda)>0$ so by continuity of $G$, there exists $\lambda>0$ such that $G(\lambda)=0$. This means that there exists a growing mode to (1.8) and this proves Theorem 1.1

\subsection{First properties of the function $G(\lambda)$ : proof of Lemma 2.1}

In this subsection, we prove Lemma 2.1, Let $\lambda \in \mathbb{R}_{+}^{*}$. Since, by assumption, the function $F^{\prime}\left(e_{0}(\theta, v)\right)$ belongs to $L^{1}(\mathbb{T} \times \mathbb{R})$, and since

$$
\forall(\theta, v) \quad\left|\int_{-\infty}^{0} \lambda e^{\lambda s} \cos (\Theta(s)) d s\right| \leqslant \int_{-\infty}^{0} \lambda e^{\lambda s} d s=1,
$$

both functions

$$
F^{\prime}\left(e_{0}(\theta, v)\right) \cos \theta \quad \text { and } \quad F^{\prime}\left(e_{0}(\theta, v)\right) \int_{-\infty}^{0} \lambda e^{\lambda s} \cos (\Theta(s, \theta, v)) d s
$$

belong to $L^{1}(\mathbb{T} \times \mathbb{R})$, so the function $f$ defined by (2.3) also belongs to $L^{1}(\mathbb{T} \times \mathbb{R})$. Hence, by integrating with respect to $\cos \theta d \theta d v$, we deduce that $G(\lambda)$ is well-defined by (2.2). The continuity of $G$ on $\mathbb{R}_{+}^{*}$ stems from dominated convergence.

Consider now a (nonzero) growing mode $e^{\lambda t} f$ of (1.8) associated to an eigenvalue $\lambda>0$. Let us prove that $G(\lambda)=0$. From $L f=\lambda f$ and (2.1), we get, in the sense of distributions,

$$
\frac{d}{d s}\left(e^{\lambda s} f(\Theta(s), V(s))\right)=e^{\lambda s} \phi_{f}^{\prime}(\Theta(s)) V(s) F^{\prime}\left(e_{0}(\Theta, V)\right) .
$$

Integrating this equation from $-R$ to 0 , we get, for almost all $(\theta, v)$ and all $R$,

$$
f(\theta, v)=e^{-\lambda R} f(\Theta(-R), V(-R))+F^{\prime}\left(e_{0}\right) \int_{-R}^{0} e^{\lambda s} \phi_{f}^{\prime}(\Theta(s)) V(s) d s,
$$

where we recall that $e_{0}(\Theta, V)=e_{0}(\theta, v)$. We multiply by a test function $\psi(\theta, v) \in$ $C_{0}^{\infty}(\mathbb{T} \times \mathbb{R})$ and integrate with respect to $(\theta, v)$,

$$
\begin{aligned}
\int_{0}^{2 \pi} \int_{\mathbb{R}} f(\theta, v) \psi(\theta, v) d \theta d v= & e^{-\lambda R} \int_{0}^{2 \pi} \int_{\mathbb{R}} f(\theta, v) \psi(\Theta(R), V(R)) d \theta d v \\
& +\int_{-R}^{0} \int_{0}^{2 \pi} \int_{\mathbb{R}} e^{\lambda s} F^{\prime}\left(e_{0}\right) \phi_{f}^{\prime}(\Theta(s)) V(s) \psi(\theta, v) d s d \theta d v
\end{aligned}
$$


In the first integral of the right-hand side, we have performed the change of variable $(\theta, v)=\left(\Theta\left(R, \theta^{\prime}, v^{\prime}\right), V\left(R, \theta^{\prime}, v^{\prime}\right)\right)$. In the second integral, we remark that $\left|\phi_{f}^{\prime}(\Theta(s))\right| \leqslant$ $\|f\|_{L^{1}}$ and, the support of $\psi$ being compact, $v$ is bounded. Hence, by $\frac{v^{2}}{2}-m_{0} \cos \theta=$ $\frac{V^{2}}{2}-m_{0} \cos \Theta, V(s)$ is bounded. Therefore, by dominated convergence (using that $\left.F^{\prime}\left(e_{0}\right) \in L^{1}\right)$, as $R \rightarrow \infty$, we get

$$
\int_{0}^{2 \pi} \int_{\mathbb{R}} f(\theta, v) \psi(\theta, v) d \theta d v=\int_{-\infty}^{0} \int_{0}^{2 \pi} \int_{\mathbb{R}} e^{\lambda s} F^{\prime}\left(e_{0}\right) \phi_{f}^{\prime}(\Theta(s)) V(s) \psi(\theta, v) d s d \theta d v
$$

i.e.

$$
\begin{aligned}
f(\theta, v) & =F^{\prime}\left(e_{0}\right) \int_{-\infty}^{0} e^{\lambda s} \phi_{f}^{\prime}(\Theta(s)) V(s) d s=F^{\prime}\left(e_{0}\right) \int_{-\infty}^{0} e^{\lambda s} \frac{d}{d s}\left(\phi_{f}(\Theta(s))\right) d s \\
& =F^{\prime}\left(e_{0}\right) \phi_{f}(\theta)-F^{\prime}\left(e_{0}\right) \int_{-\infty}^{0} \lambda e^{\lambda s} \phi_{f}(\Theta(s)) d s
\end{aligned}
$$

almost everywhere. Recall that $\phi_{f}(\theta)=-m \cos \theta$, with $m=\int_{0}^{2 \pi} \rho_{f}(\theta) \cos \theta d \theta$. Then we can rewrite this expression of $f$ as

$$
f(\theta, v)=-m F^{\prime}\left(e_{0}\right) \cos \theta-m F^{\prime}\left(e_{0}\right) \int_{-\infty}^{0} \lambda e^{\lambda s} \cos \Theta(s) d s .
$$

Integrating both sides of this equation with respect to $\cos \theta d \theta d v$ we get

$$
\begin{aligned}
m=\int_{0}^{2 \pi} \rho_{f}(\theta) \cos \theta d \theta= & -m \int_{0}^{2 \pi} \int_{\mathbb{R}} F^{\prime}\left(e_{0}\right) \cos ^{2} \theta d \theta d v \\
& +m \int_{0}^{2 \pi} \int_{\mathbb{R}} F^{\prime}\left(e_{0}\right)\left(\int_{-\infty}^{0} \lambda e^{\lambda s} \cos \Theta(s) d s\right) \cos \theta d \theta d v,
\end{aligned}
$$

i.e. $m G(\lambda)=0$. It is clear that $m \neq 0$, otherwise $f=0$ a.e.. Finally, we get $G(\lambda)=0$.

Reciprocally, assume that $G(\lambda)=0$ for some $\lambda>0$. Let $f$ be given by (2.3). We have proved above that this function belongs to $L^{1}(\mathbb{T} \times \mathbb{R})$. Moreover, since $\Theta(s,-\theta,-v)=$ $\Theta(s, \theta, v)$, we have $\int \rho_{f} \sin \theta d \theta=0$. Multiplying (2.3) by $\cos \theta$ and integrating with respect to $\theta$ and $v$, and using that $G(\lambda)=0$, we get $\int \rho_{f} \cos \theta d \theta=1$, so $f$ is not the zero function and we have $\phi_{f}(\theta)=-\cos \theta$.

We now check that the function $f$ given by (2.3) is an eigenfunction of $L$ associated with $\lambda$. From (2.3), we get

$$
f(\Theta(t), V(t))=-F^{\prime}\left(e_{0}\right) \phi_{f}(\Theta(t))+F^{\prime}\left(e_{0}\right) \int_{-\infty}^{0} \lambda e^{\lambda s} \phi_{f}(\Theta(s, \Theta(t), V(t))) d s
$$


Note that $\Theta(s, \Theta(t), V(t))=\Theta(s+t, \theta, v)$. Therefore

$$
\begin{aligned}
f(\Theta(t), V(t)) & =-F^{\prime}\left(e_{0}\right) \phi_{f}(\Theta(t))+F^{\prime}\left(e_{0}\right) \int_{-\infty}^{0} \lambda e^{\lambda s} \phi_{f}(\Theta(s+t)) d s \\
& =-F^{\prime}\left(e_{0}\right) \phi_{f}(\Theta(t))+F^{\prime}\left(e_{0}\right) e^{-\lambda t} \int_{-\infty}^{t} \lambda e^{\lambda s} \phi_{f}(\Theta(s)) d s \\
& =F^{\prime}\left(e_{0}\right) e^{-\lambda t} \int_{-\infty}^{t} e^{\lambda s} \phi_{f}^{\prime}(\Theta(s)) V(s) d s,
\end{aligned}
$$

where we integrated by parts. Then

$$
\begin{aligned}
e^{\lambda t} f(\Theta(t), V(t)) & =F^{\prime}\left(e_{0}\right) \int_{-\infty}^{t} e^{\lambda s} \phi_{f}^{\prime}(\Theta(s)) V(s) d s \\
& =\int_{-\infty}^{t} e^{\lambda s} \phi_{f}^{\prime}(\Theta(s)) \partial_{v} f_{0}(\Theta(s), V(s)) d s .
\end{aligned}
$$

Differentiating both sides with respect to $t$, we obtain in the sense of distributions that for all $t \in \mathbb{R}$,

$$
\begin{aligned}
e^{\lambda t}\left(\lambda f(\Theta(t), V(t))+V(t) \partial_{\theta} f(\Theta(t), V(t))\right. & \left.-\phi_{0}^{\prime}(\Theta(t)) \partial_{v} f(\Theta(t), V(t))\right) \\
& =e^{\lambda t} \phi_{f}^{\prime}(\Theta(t)) \partial_{v} f_{0}(\Theta(t), V(t)) .
\end{aligned}
$$

By writing this equation at $t=0$, we get $L f=\lambda f: f$ is an unstable eigenfunction of $L$. This ends the proof of Lemma 2.1.

\subsection{Limiting behavior of $G(\lambda)$ near $\lambda=0$ : proof of Lemma 2.2}

To study $\lim _{\lambda \rightarrow 0^{+}} G(\lambda)$ we need to analyze the limit of the function

$$
g_{\lambda}(\theta, v)=\int_{-\infty}^{0} \lambda e^{\lambda s} \cos \Theta(s, \theta, v) d s
$$

as $\lambda \rightarrow 0$. We provide this result in the next lemma, where we also recall some wellknown facts on the solution of the characteristics equations (2.1), which are nothing but the pendulum equations.

Lemma 2.4. Let $(\theta, v) \in \mathbb{T} \times \mathbb{R}$ and $e_{0}=\frac{v^{2}}{2}-m_{0} \cos \theta$. Consider the solution $(\Theta(s, \theta, v), V(s, \theta, v))$ to the characteristics equations (2.1). Then the following holds true.

(i) If $e_{0}>m_{0}$ then, for all $s \in \mathbb{R}$, we have

$$
\Theta\left(s+T_{e_{0}}\right)=\Theta(s)+2 \pi, \quad V\left(s+T_{e_{0}}\right)=V(s),
$$

with

$$
T_{e_{0}}=\int_{0}^{2 \pi} \frac{d \theta^{\prime}}{\sqrt{2\left(e_{0}+m_{0} \cos \theta^{\prime}\right)}}>0
$$


(ii) If $-m_{0}<e_{0}<m_{0}$ then $\Theta$ and $V$ are periodic with period given by

$$
T_{e_{0}}=4 \int_{0}^{\theta_{m_{0}}} \frac{d \theta}{\sqrt{2\left(e_{0}+m_{0} \cos \theta\right)}}=\frac{4}{\sqrt{m_{0}}} \int_{0}^{\pi / 2} \frac{d \theta}{\sqrt{1-\frac{m_{0}+e_{0}}{2 m_{0}} \sin ^{2} \theta}}>0
$$

where $\theta_{m_{0}}=\arccos \left(-\frac{e_{0}}{m_{0}}\right)$.

(iii) We have

$$
\lim _{\lambda \rightarrow 0^{+}} g_{\lambda}(\theta, v)= \begin{cases}\frac{1}{T_{e_{0}}} \int_{0}^{2 \pi} \frac{\cos \theta^{\prime}}{\sqrt{2\left(e_{0}+m_{0} \cos \theta^{\prime}\right)}} d \theta^{\prime} & \text { if } e_{0}>m_{0}, \\ \frac{4}{T_{e_{0}}} \int_{0}^{\theta_{m_{0}}} \frac{\cos \theta^{\prime}}{\sqrt{2\left(e_{0}+m_{0} \cos \theta^{\prime}\right)}} d \theta^{\prime} & \text { if }-m_{0}<e_{0}<m_{0} .\end{cases}
$$

Proof. (i) Let $e_{0}>m_{0}$. Without loss of generality, since $\Theta(s,-\theta,-v)=\Theta(s, \theta, v)$ and $V(s,-\theta,-v)=V(s, \theta, v)$, we can only treat the case $v>0$. As we have

$$
\frac{V(s)^{2}}{2}=e_{0}+m_{0} \cos \Theta(s) \geqslant e_{0}-m_{0}>0,
$$

$V(s)$ does not vanish and remains positive. Hence $\Theta(s)$ is the solution of the following autonomous equation

$$
\dot{\Theta}(s)=V(s)=\sqrt{2\left(e_{0}+m_{0} \cos \Theta(s)\right)}
$$

and is strictly increasing with $\Theta(s) \rightarrow+\infty$ as $s \rightarrow+\infty$. Let $T_{e_{0}}$ be the unique time such that $\Theta\left(T_{e_{0}}\right)=\theta+2 \pi$. By Cauchy-Lipschitz's theorem, we have $\Theta\left(s+T_{e_{0}}\right)-2 \pi=\Theta(s)$ and (2.7) holds. Defining

$$
P(\tau)=\int_{0}^{\tau} \frac{d \theta^{\prime}}{\sqrt{2\left(e_{0}+m_{0} \cos \theta^{\prime}\right)}}
$$

the solution of (2.11) satisfies $P(\Theta(s))-P(\theta)=s$. Therefore, we have $T_{e_{0}}=P(\theta+$ $2 \pi)-P(\theta)$, from which we get (2.8).

(ii) Let $-m_{0}<e_{0}<m_{0}$. In this case, $\Theta(s)$ will oscillate between the two values $\theta_{m_{0}}=\arccos \left(-\frac{e_{0}}{m_{0}}\right)$ and $-\theta_{m_{0}}$ with a period $T_{e_{0}}$ given by (2.9). On the half-periods where $\Theta$ is increasing, we also have (2.11). We skip the details of the proof, which is classical.

(iii) We remark that $\cos \Theta\left(s+k T_{e_{0}}\right)=\cos \Theta(s)$ for all $s \in \mathbb{R}$ and $k \in \mathbb{Z}$. Indeed, by (i), for $e_{0}>m_{0}$ we have $\Theta\left(s+k T_{e_{0}}\right)=\Theta(s)+2 \pi k$ and, by (ii), for $-m_{0}<e_{0}<m_{0}$ we have 
$\Theta\left(s+k T_{e_{0}}\right)=\Theta(s)$. Hence, we compute from (2.6)

$$
\begin{aligned}
g_{\lambda}(\theta, v) & =\sum_{k=0}^{+\infty} \int_{k T_{e_{0}}}^{(k+1) T_{e_{0}}} \lambda e^{-\lambda s} \cos \Theta(-s) d s \\
& =\sum_{k=0}^{+\infty} \int_{0}^{T_{e_{0}}} \lambda e^{-\lambda s-k \lambda T_{e_{0}}} \cos \Theta(-s) d s \\
& =\left(\sum_{k=0}^{+\infty} e^{-k \lambda T_{e_{0}}}\right) \int_{0}^{T_{e_{0}}} \lambda e^{-\lambda s} \cos \Theta(-s) d s \\
& =\frac{\lambda}{1-e^{-\lambda T_{e_{0}}}} \int_{0}^{T_{e_{0}}} e^{-\lambda s} \cos \Theta(-s) d s .
\end{aligned}
$$

Therefore, clearly,

$$
\lim _{\lambda \rightarrow 0^{+}} g_{\lambda}(\theta, v)=\frac{1}{T_{e_{0}}} \int_{0}^{T_{e_{0}}} \cos \Theta(-s) d s=\frac{1}{T_{e_{0}}} \int_{0}^{T_{e_{0}}} \cos \Theta(s) d s .
$$

If $e_{0}>m_{0}$, we perform the change of variable $\theta^{\prime}=\Theta(s)$ which is strictly increasing from $\left[0, T_{e_{0}}\right]$ to $[\theta, \theta+2 \pi]$. Using (2.11), we obtain

$$
\lim _{\lambda \rightarrow 0^{+}} g_{\lambda}(\theta, v)=\frac{1}{T_{e_{0}}} \int_{\theta}^{\theta+2 \pi} \frac{\cos \theta^{\prime}}{\sqrt{2\left(e_{0}+m_{0} \cos \theta^{\prime}\right)}} d \theta^{\prime}=\frac{1}{T_{e_{0}}} \int_{0}^{2 \pi} \frac{\cos \theta^{\prime}}{\sqrt{2\left(e_{0}+m_{0} \cos \theta^{\prime}\right)}} d \theta^{\prime} .
$$

If $-m_{0}<e_{0}<m_{0}$, we can always choose a time $t_{0}$ such that $\Theta\left(t_{0}\right)=-\theta_{m_{0}}, \Theta\left(t_{0}+\right.$ $\left.T_{e_{0}} / 2\right)=\theta_{m_{0}}, \Theta(s)$ is strictly increasing on $\left[t_{0}, t_{0}+T_{e_{0}} / 2\right]$ and such that $\Theta(s)=\Theta\left(2 t_{0}+\right.$ $\left.T_{e_{0}}-s\right)$ for $s \in\left[t_{0}+T_{e_{0}} / 2, t_{0}+T_{e_{0}}\right]$. We have

$$
\begin{aligned}
\lim _{\lambda \rightarrow 0^{+}} g_{\lambda}(\theta, v) & =\frac{1}{T_{e_{0}}} \int_{t_{0}}^{t_{0}+T_{e_{0}} / 2} \cos \Theta(s) d s+\frac{1}{T_{e_{0}}} \int_{t_{0}+T_{e_{0}} / 2}^{t_{0}+T_{e_{0}}} \cos \Theta(s) d s \\
& =\frac{1}{T_{e_{0}}} \int_{t_{0}}^{t_{0}+T_{e_{0}} / 2} \cos \Theta(s) d s+\frac{1}{T_{e_{0}}} \int_{t_{0}+T_{e_{0}} / 2}^{t_{0}+T_{e_{0}}} \cos \Theta\left(2 t_{0}+T_{e_{0}}-s\right) d s \\
& =\frac{2}{T_{e_{0}}} \int_{t_{0}}^{t_{0}+T_{e_{0}} / 2} \cos \Theta(s) d s \\
& =\frac{2}{T_{e_{0}}} \int_{-\theta_{m_{0}}}^{\theta_{m_{0}}} \frac{\cos \theta^{\prime}}{\sqrt{2\left(e_{0}+m_{0} \cos \theta^{\prime}\right)}} d \theta^{\prime}=\frac{4}{T_{e_{0}}} \int_{0}^{\theta_{m_{0}}} \frac{\cos \theta^{\prime}}{\sqrt{2\left(e_{0}+m_{0} \cos \theta^{\prime}\right)}} d \theta^{\prime}
\end{aligned}
$$

where, on the time interval $\left[t_{0}, t_{0}+T_{e_{0}} / 2\right]$, we performed the change of variable $\theta^{\prime}=$ $\Theta(s)$.

Proof of Lemma 2.2. Now we come back to the definition (2.2) of $G_{\lambda}$, which reads

$$
\begin{aligned}
G(\lambda)=1 & +\int_{0}^{2 \pi} \int_{\mathbb{R}} F^{\prime}\left(\frac{v^{2}}{2}-m_{0} \cos \theta\right) \cos ^{2} \theta d \theta d v \\
& -\int_{0}^{2 \pi} \int_{\mathbb{R}} F^{\prime}\left(\frac{v^{2}}{2}-m_{0} \cos \theta\right) g_{\lambda}(\theta, v) \cos \theta d \theta d v .
\end{aligned}
$$


We remark that $\left|g_{\lambda}(\theta, v)\right| \leqslant 1$ and recall that the function $F^{\prime}\left(\frac{v^{2}}{2}-m_{0} \cos \theta\right)$ belongs to $L^{1}(\mathbb{T} \times \mathbb{R})$. Therefore, we can pass to the limit in the second integral by dominated convergence and deduce from Lemma 2.4 (iii) (note that the set $\left\{(\theta, v): e_{0}(\theta, v) \leqslant-m_{0}\right\}$ is of measure zero) that

$$
\begin{aligned}
\lim _{\lambda \rightarrow 0^{+}} G(\lambda)=1 & +\int_{0}^{2 \pi} \int_{\mathbb{R}} F^{\prime}\left(e_{0}\right) \cos ^{2} \theta d \theta d v \\
& -\iint_{e_{0}(\theta, v)>m_{0}} F^{\prime}\left(e_{0}\right)\left(\frac{1}{T_{e}} \int_{0}^{2 \pi} \frac{\cos \theta^{\prime}}{\sqrt{2\left(e_{0}+m_{0} \cos \theta^{\prime}\right)}} d \theta^{\prime}\right) \cos \theta d \theta d v \\
& -\iint_{-m_{0}<e_{0}(\theta, v)<m_{0}} F^{\prime}\left(e_{0}\right)\left(\frac{4}{T_{e}} \int_{0}^{\theta_{m_{0}}} \frac{\cos \theta^{\prime}}{\sqrt{2\left(e_{0}+m_{0} \cos \theta^{\prime}\right)}} d \theta^{\prime}\right) \cos \theta d \theta d v \\
=1+ & \int_{0}^{2 \pi} \int_{\mathbb{R}} F^{\prime}\left(e_{0}\right) \cos ^{2} \theta d \theta d v \\
& -\int_{0}^{2 \pi} \int_{\mathbb{R}} F^{\prime}\left(e_{0}\right)\left(\frac{\int_{\mathcal{D}_{e_{0}}} \cos \theta^{\prime}\left(e_{0}+m_{0} \cos \theta^{\prime}\right)^{-1 / 2} d \theta^{\prime}}{\int_{\mathcal{D}_{e_{0}}}\left(e_{0}+m_{0} \cos \theta^{\prime}\right)^{-1 / 2} d \theta^{\prime}}\right) \cos \theta d \theta d v \\
=1+ & \int_{0}^{2 \pi} \int_{\mathbb{R}} F^{\prime}\left(e_{0}\right) \cos (\theta)^{2} d v d \theta-\int_{0}^{2 \pi} \int_{\mathbb{R}} F^{\prime}\left(e_{0}\right)\left(\Pi_{m_{0}}(\cos \theta)\right)^{2} d \theta d v,
\end{aligned}
$$

with

$$
\left(\Pi_{m_{0}} h\right)(e)=\frac{\int_{\mathcal{D}_{e}}\left(e+m_{0} \cos \theta\right)^{-1 / 2} h(\theta) d \theta}{\int_{\mathcal{D}_{e}}\left(e+m_{0} \cos \theta\right)^{-1 / 2} d \theta},
$$

for all function $h(\theta)$ and

$$
\mathcal{D}_{e}=\left\{\theta^{\prime} \in \mathbb{T}: m_{0} \cos \theta^{\prime}>-e\right\} .
$$

Here the operator $\Pi_{m_{0}}$ is a variant of the operator $\Pi$ given by (3.8) in [21], this operator should be understood as the "projector" onto the functions which depend only on the microscopic energy $e_{0}(\theta, v)$. A projector of this type is also mentioned in the work by Guo and Lin [15].

Now we remark that straightforward calculations give

$$
\begin{aligned}
1-\kappa_{0} & =1+\int_{0}^{2 \pi} \int_{\mathbb{R}} F^{\prime}\left(e_{0}\right)\left(\cos ^{2} \theta-2 \cos \theta \Pi_{m_{0}}(\cos \theta)+\left(\Pi_{m_{0}}(\cos \theta)\right)^{2}\right) d \theta d v \\
& =1+\int_{0}^{2 \pi} \int_{\mathbb{R}} F^{\prime}\left(e_{0}\right) \cos ^{2} \theta d \theta d v-\iint F^{\prime}\left(e_{0}\right)\left(\Pi_{m_{0}}(\cos \theta)\right)^{2} d \theta d v
\end{aligned}
$$


where

$$
-\kappa_{0}:=\int_{0}^{2 \pi} \int_{\mathbb{R}} F^{\prime}\left(e_{0}\right)\left(\frac{\int_{\mathcal{D}_{e}}\left(\cos \theta-\cos \theta^{\prime}\right)\left(e_{0}+m_{0} \cos \theta^{\prime}\right)^{-1 / 2} d \theta^{\prime}}{\int_{\mathcal{D}_{e}}\left(e_{0}+m_{0} \cos \theta^{\prime}\right)^{-1 / 2} d \theta^{\prime}}\right)^{2} d \theta d v
$$

This calculation uses that $\Pi_{m_{0}}$ is a projector. We finally get (2.4) and the proof of Lemma 2.2 is complete.

\subsection{Limiting behavior of $G(\lambda)$ as $\lambda \rightarrow \infty$ : proof of Lemma 2.3}

In this subsection, we prove Lemma 2.3. An integration by parts in (2.6) yields

$$
g_{\lambda}(\theta, v)=\cos \theta+\int_{-\infty}^{0} e^{\lambda s} V(s, \theta, v) \sin \Theta(s, \theta, v) d s .
$$

The velocity can be bounded independently of $s$ thanks to the conservation of the energy,

$$
|V(s)|=\left(v^{2}+2 m_{0} \cos \theta-2 m_{0} \cos \Theta(s)\right)^{1 / 2} \leqslant\left(v^{2}+4 m_{0}\right)^{1 / 2} .
$$

Thus

$$
\left|\int_{-\infty}^{0} e^{\lambda s} \sin \Theta(s, \theta, v) V(s, \theta, v) d s\right| \leqslant\left(v^{2}+4 m_{0}\right)^{1 / 2} \int_{-\infty}^{0} e^{\lambda s} d s=\frac{\left(v^{2}+4 m_{0}\right)^{1 / 2}}{\lambda}
$$

and, for all $(\theta, v)$,

$$
\lim _{\lambda \rightarrow+\infty} g_{\lambda}(\theta, v)=\cos \theta .
$$

Using again that $\left|g_{\lambda}(\theta, v)\right| \leqslant 1$ and that $F^{\prime}\left(e_{0}(\theta, v)\right)$ belongs to $L^{1}$, we deduce directly (2.5) from (2.12) and from dominated convergence. The proof of Lemma 2.3 is complete.

\section{A nonlinear instability result: proof of Theorem 1.2}

We start by an analysis of the linearized HMF operator $L$ around the inhomogeneous equilibrium state $f_{0}$, where $L$ is given by (1.9). We write

$$
L=L_{0}+K
$$

where

$$
L_{0} f=-v \partial_{\theta} f-E_{f_{0}} \partial_{v} f, \quad K f=-E_{f} \partial_{v} f_{0}, \quad E_{f}=-\partial_{\theta} \phi_{f}
$$




\subsection{Estimates on the semigroup $e^{t L}$}

Let us state some useful properties of the operator $L_{0}$ given by (3.2). Since $\phi_{0}(\theta)=$ $-m_{0} \cos \theta$ is smooth, the characteristics equations (2.1) admit a unique solution $\Theta(s, \theta, v), V(s, \theta, v)$, which is globally defined and $\mathcal{C}^{\infty}$ in the variables $(s, \theta, v)$. Moreover, this solution has bounded derivatives with respect to $\theta$ and $v$, locally in time. Let $k \in \mathbb{N}$. For any $f$ in the Sobolev space $W^{k, 1}(\mathbb{T} \times \mathbb{R})$, the function

$$
e^{t L_{0}} f(t, \theta, v):=f(\Theta(-t, \theta, v), V(-t, \theta, v)), \quad \forall t \geqslant 0,
$$

belongs to $\mathcal{C}^{0}\left(\mathbb{R}, W^{k, 1}(\mathbb{T} \times \mathbb{R})\right)$ and is clearly a solution to $\partial_{t} g=L_{0} g$ with initial data $f$. This means that the semigroup $e^{t L_{0}}$ generated by the operator $L_{0}$ is strongly continuous on $W^{k, 1}(\mathbb{T} \times \mathbb{R})$.

Our aim is to apply the abstract results in [25] concerning perturbation theory of linear operators. Hence, we need to prove the following estimate. For all $\beta>0$, there exists a positive constant $M_{\beta, k}$ such that

$$
\left\|e^{t L_{0}} f\right\|_{W^{k, 1}} \leqslant M_{\beta, k} e^{t \beta}\|f\|_{W^{k, 1}} \quad \forall f \in W^{k, 1}(\mathbb{T} \times \mathbb{R}), \quad \forall t \geqslant 0,
$$

where $M_{\beta, k}$ depends on $\beta$ and $k$. In fact this estimate will be proved for a subclass of functions $f$. From the assumptions of Theorem 1.2 on $F$, there exists $e_{*}<m_{0}$ such that support of $F$ is $\left(-\infty, e_{*}\right]$. This means that the support of $f_{0}$ is contained in $\overline{\Omega_{0}}$, where $\Omega_{0}$ is the smooth open set

$$
\Omega_{0}=\left\{(\theta, v): \frac{v^{2}}{2}-m_{0} \cos \theta<e_{*}\right\} .
$$

We then introduce the functional space

$$
\mathscr{E}_{k}=\left\{f \in W^{k, 1}(\mathbb{T} \times \mathbb{R}): \quad \operatorname{Supp}(f) \subset \overline{\Omega_{0}}\right\},
$$

and claim that for all $f \in \mathscr{E}_{k}$, we have $e^{t L_{0}} f \in \mathscr{E}_{k}$ and, for all $\beta>0$,

$$
\left\|e^{t L_{0}} f\right\|_{W^{k, 1}} \leqslant M_{\beta, k} e^{t \beta}\|f\|_{W^{k, 1}}, \quad \forall f \in \mathscr{E}_{k}, \quad \forall t \geqslant 0
$$

$M_{\beta, k}$ being a positive constant depending on $\beta$ and $k$.

Assume for the moment that estimate (3.5) holds true. From the assumptions of Theorem 1.2, one deduces that $\partial_{v} f_{0} \in \mathscr{E}_{k}$. It is then easy to check that $e^{t L_{0}} K$ is a compact operator on $\mathscr{E}_{k}$, for all $t \in \mathbb{R}$, and the map $t \mapsto e^{t L_{0}} K \in \mathscr{L}\left(\mathscr{E}_{k}\right)$ is continuous on $\mathbb{R}$. Hence, $K$ is $L_{0}$-smoothing in the sense of [25] (page 707). Assumptions of Theorem 1.1 in 25] are therefore satisfied, which implies that $L$ generates a strongly continuous semigroup $e^{t L}$. Now, from Theorem 1.2 in [25], for all $\beta>0$, any point of the spectrum $\sigma(L)$ lying in the half plane $\operatorname{Re} z>\beta$ is an isolated eigenvalue with finite algebraic multiplicity. Furthermore, the set $\sigma(L) \cap\{\operatorname{Re} z>\beta\}$ is finite.

The assumptions of Theorem 1.2 clearly imply those of Theorem 1.1. Hence $L$ admits at least one eigenvalue $\lambda \in \mathbb{R}_{+}^{*}$ associated with an eigenfunction $\tilde{f} \in L^{1}(\mathbb{T} \times \mathbb{R})$. We claim 
that, in fact, $\tilde{f} \in \mathscr{E}_{k}$ which will be proved below. This means that the set of eigenvalues of $L$ on $\mathscr{E}_{k}$ with positive real part is not empty, and we therefore can choose an eigenvalue $\gamma$ with positive maximal real part. Finally, we apply Theorem 1.3 in [25] and get that, for all $\beta>\operatorname{Re} \gamma$, there exists a positive constant $M_{\beta, k}$ such that

$$
\left\|e^{t L} f\right\|_{W^{k, 1}} \leqslant M_{\beta, k} e^{t \beta}\|f\|_{W^{k, 1}} \quad \forall f \in \mathscr{E}_{k}, \quad \forall t \geqslant 0 .
$$

Proof of (3.5) and of the claim $\tilde{f} \in \mathscr{E}_{k}$. Let $f \in \mathscr{E}_{k}$. From (3.3), we clearly have

$$
\left\|e^{t L_{0}} f\right\|_{L^{1}}=\|f\|_{L^{1}}, \quad \forall f \in L^{1}, \quad \forall t \geqslant 0 .
$$

Moreover, we know from the analysis of the characteristics problem (2.1) performed in Section 2, that by conservation of the energy, for all $(\theta, v) \in \Omega_{0}$, we have $(\Theta(t, \theta, v), V(t, \theta, v)) \in \Omega_{0}$. Thus

$$
\operatorname{Supp}\left(e^{t L_{0}} f\right) \subset \overline{\Omega_{0}} .
$$

Let $k \geqslant 1$. By (3.3), to get an estimate of $e^{t L_{0}} f$ in $W^{k, 1}(\mathbb{T} \times \mathbb{R})$, it is sufficient to estimate $\Theta$ and $V$ in $W^{k, \infty}(\mathbb{T} \times \mathbb{R})$ for $(\theta, v) \in \Omega_{0}$. Recall that, since $e_{*}<m_{0}, \Theta$ and $V$ are periodic functions with period $T_{e_{0}}$. Moreover, (2.9) shows that $T_{e_{0}}$ is a $\mathcal{C}^{\infty}$ function of $e_{0}$ on $\left[-m_{0}, e_{*}\right]$, which means that it is also a $\mathcal{C}^{\infty}$ function of $(\theta, v)$. Note also that $\frac{2 \pi}{\sqrt{m_{0}}} \leqslant T_{e_{0}} \leqslant T_{e_{*}}$. Define now the following 1-periodic functions

$$
\widetilde{\Theta}(s, \theta, v)=\Theta\left(s T_{e_{0}}, \theta, v\right), \quad \tilde{V}(s, \theta, v)=V\left(s T_{e_{0}}, \theta, v\right)
$$

satisfying

$$
\frac{d \widetilde{\Theta}}{d s}=T_{e_{0}} \widetilde{V}, \quad \frac{d \tilde{V}}{d s}=-m_{0} T_{e_{0}} \sin \widetilde{\Theta}
$$

Applying Gronwall Lemma, one gets

$$
\left|\partial_{s}^{r} \partial_{\theta}^{j} \partial_{v}^{\ell} \widetilde{\Theta}\right|+\left|\partial_{s}^{r} \partial_{\theta}^{j} \partial_{v}^{\ell} \tilde{V}\right| \leqslant C_{k} e^{C_{k} s}, \quad \forall s \geqslant 0, \quad \forall(\theta, v) \in \Omega_{0}, \quad \text { for } r+j+\ell \leqslant k .
$$

The period of $\widetilde{\Theta}$ and $\tilde{V}$ being independent of $(\theta, v)$, the functions $\partial_{\theta}^{j} \partial_{v}^{\ell} \widetilde{\Theta}$ and $\partial_{\theta}^{j} \partial_{v}^{\ell} \tilde{V}$ are also 1-periodic and therefore

$$
\left|\partial_{s}^{r} \partial_{\theta}^{j} \partial_{v}^{\ell} \widetilde{\Theta}\right|+\left|\partial_{s}^{r} \partial_{\theta}^{j} \partial_{v}^{\ell} \widetilde{V}\right| \leqslant C_{k}^{\prime}=C_{k} e^{C_{k}}, \quad \forall s \geqslant 0, \quad \forall(\theta, v) \in \Omega_{0}, \quad \text { for } r+j+\ell \leqslant k .
$$

Coming back to $\Theta$ and $V$, we deduce

$$
\left|\partial_{\theta}^{j} \partial_{v}^{\ell} \Theta\right|+\left|\partial_{\theta}^{j} \partial_{v}^{\ell} V\right| \leqslant C_{k}\left(1+s^{k}\right), \quad \forall s \geqslant 0, \quad \forall(\theta, v) \in \Omega_{0}, \quad \text { for } j+\ell \leqslant k .
$$

Using this estimate and (3.3), we finally get (3.5)).

Let us finally prove that $\tilde{f} \in \mathscr{E}_{k}$. By Lemma 2.1, the function $\tilde{f}$ is given by

$$
\tilde{f}(\theta, v)=-F^{\prime}\left(e_{0}(\theta, v)\right) \cos \theta+F^{\prime}\left(e_{0}(\theta, v)\right) \int_{-\infty}^{0} \lambda e^{\lambda s} \cos (\Theta(s, \theta, v)) d s .
$$


Hence, the support of $F^{\prime}\left(e_{0}(\theta, v)\right)$ being in $\overline{\Omega_{0}}$, the support on $\tilde{f}$ will also be contained in $\overline{\Omega_{0}}$. Moreover, by using (3.7), we obtain that, for some $C_{k}>0$, we have

$$
\forall j+\ell \leqslant k, \quad \forall(\theta, v) \in \mathbb{T} \times \mathbb{R}, \quad\left|\partial_{\theta}^{j} \partial_{v}^{\ell} \int_{-\infty}^{0} \lambda e^{\lambda s} \cos (\Theta(s, \theta, v)) d s\right| \leqslant C_{k} .
$$

This is sufficient to deduce from $F \in \mathcal{C}^{\infty}$ that $\tilde{f} \in \mathscr{E}_{k}$.

\subsection{An iterative scheme}

In this part, we prove Theorem 1.2 by following the strategy developed by Grenier in [14, which has been also used in [17, 18] to analyse instabilities for homogeneous steady states of Vlasov-Poisson models. Let $N \geqslant 1$ be an integer to be fixed later. According to the previous subsection, we can consider an eigenvalue $\gamma$ of $L$ on $\mathscr{E}_{N}$ with maximal real part, $\operatorname{Re} \gamma>0$. Let $g \in \mathscr{E}_{N}$ be an associated eigenfunction. With no loss of generality, we may assume that $\|\operatorname{Re} g\|_{L^{1}}=1$. Let

$$
f_{1}(t, \theta, v)=\operatorname{Re}\left(e^{\gamma t} g(\theta, v)\right) \chi_{\delta}\left(e_{0}(\theta, v)\right),
$$

with $e_{0}(\theta, v)=\frac{v^{2}}{2}+\phi_{0}(\theta)$ and where $0 \leqslant \chi_{\delta}(e) \leqslant 1$ is a smooth real-valued truncation function to be defined further, in order to ensure the positivity of $f_{0}+\delta f_{1}(0)$. Note that $f_{1}$ is almost a growing mode solution to the linearized HMF model (1.8) since we have

$$
\left(\partial_{t}-L\right) f_{1}=\operatorname{Re}\left(e^{\gamma t} \widetilde{R}_{\delta}\right),
$$

where

$$
\widetilde{R}_{\delta}=\left(-E_{\left(1-\chi_{\delta}\right) g}+\left(1-\chi_{\delta}\right) E_{g}\right) \partial_{v} f_{0}
$$

will be small. We now construct an approximate solution $f_{a p p}^{N}$ to the HMF model (1.1) of the form

$$
f_{a p p}^{N}=f_{0}+\sum_{k=1}^{N} \delta^{k} f_{k},
$$

for sufficiently small $\delta>0$, in which $f_{k}(k \geqslant 2)$ solves inductively the linear problem

$$
\left(\partial_{t}-L\right) f_{k}+\sum_{j=1}^{k-1} E_{f_{j}} \partial_{v} f_{k-j}=0
$$

with $f_{k}(0)=0$. Then $f_{a p p}^{N}$ approximately solves the HMF model (1.1) in the sense that

$$
\partial_{t} f_{a p p}^{N}+v \partial_{\theta} f_{a p p}^{N}-\partial_{\theta} \phi_{f_{a p p}^{N}} \partial_{v} f_{a p p}^{N}=R_{N}+\delta \operatorname{Re}\left(e^{\gamma t} \widetilde{R}\right),
$$

where the remainder term $R_{N}$ is given by

$$
R_{N}=\sum_{1 \leqslant j, \ell \leqslant N ; j+\ell \geqslant N+1} \delta^{j+\ell} E_{f_{j}} \partial_{v} f_{\ell} .
$$


Step 1. Estimate of $f_{k}$. We claim that $f_{k} \in \mathscr{E}_{N-k+1}$ and, for all $1 \leqslant k \leqslant N$,

$$
\left\|f_{k}\right\|_{W^{N-k+1,1}} \leqslant C_{k} e^{k t \operatorname{Re} \gamma} .
$$

We proceed by induction. From (3.8), this estimate is a consequence, for $k=1$, of

$$
\left\|g \chi_{\delta}\right\|_{W^{N, 1}} \leqslant C_{1},
$$

which is proved below in Step 5 . Let $k \geqslant 2$. We have

$$
f_{k}(t)=-\int_{0}^{t} e^{L(t-s)} \sum_{j=1}^{k-1} E_{f_{j}}(s) \partial_{v} f_{k-j}(s) d s .
$$

Therefore, for $\operatorname{Re} \gamma<\beta<2 \operatorname{Re} \gamma$,

$$
\begin{aligned}
\left\|f_{k}\right\|_{W^{N-k+1,1}} \leqslant & \sum_{j=1}^{k-1} \int_{0}^{t}\left\|e^{L(t-s)}\left(E_{f_{j}}(s) \partial_{v} f_{k-j}(s)\right)\right\|_{W^{N-k+1,1}} d s \\
\leqslant & M_{\beta, N-k+1} \sum_{j=1}^{k-1} \int_{0}^{t} e^{\beta(t-s)}\left\|E_{f_{j}}(s)\right\|_{W^{N-k+1, \infty}}\left\|\partial_{v} f_{k-j}(s)\right\|_{W^{N-k+1,1}} d s \\
\leqslant & M_{\beta, N-k+1} \sum_{j=1}^{k-1} \int_{0}^{t} e^{\beta(t-s)}\left\|f_{j}(s)\right\|_{L^{1}}\left\|f_{k-j}(s)\right\|_{W^{N-k+2,1}} d s \\
& \quad \operatorname{since} k-j \leqslant k-1, \\
\leqslant & M_{\beta, N-k+1}\left(\sum_{j=1}^{k-1} C_{j} C_{k-j}\right) \int_{0}^{t} e^{\beta(t-s)} e^{k s \operatorname{Re} \gamma} d s \\
\leqslant & \frac{M_{\beta, N-k+1}}{k \operatorname{Re} \gamma-\beta}\left(\sum_{j=1}^{k-1} C_{j} C_{k-j}\right) e^{k t \operatorname{Re} \gamma},
\end{aligned}
$$

where we used (3.6) and the recursive assumption. This ends the proof of (3.14).

Step 2. Estimates of $f_{a p p}^{N}-f_{0}$ and $R_{N}$. The parameter $\delta$ and the time $t$ will be such that

$$
\delta e^{t \operatorname{Re} \gamma} \leqslant \min \left(\frac{1}{2}, \frac{1}{2 K_{N}}\right), \quad K_{N}=\max _{1 \leqslant k \leqslant N} C_{k} .
$$

Hence, from (3.14) we obtain

$$
\left\|f_{a p p}^{N}-f_{0}\right\|_{W^{1,1}} \leqslant \sum_{k=1}^{N} \delta^{k} C_{k} e^{k t \operatorname{Re} \gamma} \leqslant K_{N} \frac{\delta e^{t \operatorname{Re} \gamma}}{1-\delta e^{t \operatorname{Re} \gamma}} \leqslant 1
$$

and

$$
\left\|R_{N}\right\|_{L^{1}} \leqslant \sum_{k=N+1}^{+\infty} \delta^{k} e^{k t \operatorname{Re} \gamma} \sum_{1 \leqslant j, \ell \leqslant N ; j+\ell=k} C_{j} C_{\ell} \leqslant \widetilde{C}_{N}\left(\delta e^{t \operatorname{Re} \gamma}\right)^{N+1}
$$


Step 3. Estimate of $f-f_{a p p}^{N}$. Let $f(t)$ be the solution of (1.1) with initial data $f_{0}+$ $\delta \operatorname{Re} g \chi_{\delta}$ and let $h=f-f_{a p p}^{N}$. Note that the positivity of $f(t)$ is ensured by $f_{0}+\delta \operatorname{Re} g \chi_{\delta} \geqslant$ 0 and that we have

$$
\left\|f(0)-f_{0}\right\|_{L^{1}} \leqslant \delta
$$

The function $h$ satisfies the following equation

$$
\partial_{t} h+v \partial_{\theta} h+E_{f} \partial_{v} h=\left(E_{f_{a p p}^{N}}-E_{f}\right) \partial_{v} f_{a p p}^{N}-R_{N}-\delta \operatorname{Re}\left(e^{\gamma t} \widetilde{R}_{\delta}\right)
$$

with $h(0)=0$. To get a $L^{1}$-estimate of $h$, we multiply this equation by $\operatorname{sign}(h)$ and integrate in $(\theta, v)$. We get

$$
\begin{aligned}
\frac{d}{d t}\|h\|_{L^{1}} & \leqslant\left\|E_{f_{a p p}^{N}}-E_{f}\right\|_{L^{\infty}}\left\|\partial_{v} f_{a p p}^{N}\right\|_{L^{1}}+\left\|R_{N}\right\|_{L^{1}}+\delta e^{t \operatorname{Re} \gamma}\left\|\widetilde{R}_{\delta}\right\|_{L^{1}} \\
& \leqslant\|h\|_{L^{1}}\left\|\partial_{v} f_{a p p}^{N}\right\|_{L^{1}}+\left\|R_{N}\right\|_{L^{1}}+\delta e^{t \operatorname{Re} \gamma}\left\|\widetilde{R}_{\delta}\right\|_{L^{1}} .
\end{aligned}
$$

From Step 2 we have $\left\|\partial_{v} f_{a p p}^{N}\right\|_{L^{1}} \leqslant\left\|\partial_{v} f_{0}\right\|_{L^{1}}+1$, which implies that

$$
\|h(t)\|_{L^{1}} \leqslant \int_{0}^{t} e^{(t-s)\left(\left\|\partial_{v} f_{0}\right\|_{L^{1}}+1\right)}\left(\left\|R_{N}(s)\right\|_{L^{1}}+\delta e^{s \operatorname{Re} \gamma}\left\|\widetilde{R}_{\delta}\right\|_{L^{1}}\right) d s .
$$

Again from Step 2, we then get

$$
\|h(t)\|_{L^{1}} \leqslant \int_{0}^{t} e^{(t-s)\left(\left\|\partial_{v} f_{0}\right\|_{L^{1}}+1\right)}\left(\widetilde{C}_{N}\left(\delta e^{s \operatorname{Re} \gamma}\right)^{N+1}+\delta e^{s \operatorname{Re} \gamma}\left\|\widetilde{R}_{\delta}\right\|_{L^{1}}\right) d s
$$

We now fix $N$ as follows (with the notation $\lfloor\cdot\rfloor$ for the integer function)

$$
N:=\left\lfloor\frac{\left\|\partial_{v} f_{0}\right\|_{L^{1}}+1}{\operatorname{Re} \gamma}\right\rfloor+1 \geqslant 1
$$

and claim that $\chi$ may be chosen such that

$$
\left\|\widetilde{R}_{\delta}\right\|_{L^{1}} \leqslant\left(\delta e^{s \operatorname{Re} \gamma}\right)^{N}
$$

see Step 5 for the proof. This yields

$$
\left\|f-f_{a p p}^{N}\right\|_{L^{1}}(t) \leqslant \check{C}_{N}\left(\delta e^{t \operatorname{Re} \gamma}\right)^{N+1}
$$

with $\check{C}_{N}=\frac{1+\widetilde{C}_{N}}{3 \operatorname{Re} \gamma}$.

Step 4. End of the proof. Since Re $g$ is not zero, we can choose a real valued function $\varphi(\theta, v)$ in $L^{\infty}$ such that $\|\varphi\|_{L^{\infty}}=1$ and

$$
\operatorname{Re} z_{g}>0 \text { with } z_{g}=\int_{0}^{2 \pi} \int_{\mathbb{R}} g \varphi d \theta d v .
$$


Denoting

$$
z_{g, \delta}=\int_{0}^{2 \pi} \int_{\mathbb{R}} g \chi_{\delta} \varphi d \theta d v
$$

we have

$$
\begin{aligned}
\iint f_{1} \varphi d \theta d v & =e^{t \operatorname{Re} \gamma} \operatorname{Re}\left(e^{i t \operatorname{Im} \gamma} z_{g, \delta}\right) \\
& \geqslant e^{t \operatorname{Re} \gamma} \operatorname{Re}\left(e^{i t \operatorname{Im} \gamma} z_{g}\right)-e^{t \operatorname{Re} \gamma}\left|z_{g}-z_{g, \delta}\right| \\
& \geqslant e^{t \operatorname{Re} \gamma} \operatorname{Re}\left(e^{i t \operatorname{Im} \gamma} z_{g}\right)-e^{t \operatorname{Re} \gamma}\left\|g\left(1-\chi_{\delta}\right)\right\|_{L^{1}}
\end{aligned}
$$

We claim that

$$
\lim _{\delta \rightarrow 0}\left\|\left(1-\chi_{\delta}\right) g\right\|_{L^{1}}=0,
$$

which again will be proved in Step 5. In order to end the proof of Theorem 1.2, we estimate from below, using (3.18) and (3.14),

$$
\begin{aligned}
\left\|f-f_{0}\right\|_{L^{1}} & \geqslant \iint\left(f-f_{0}\right) \varphi d \theta d v=\iint\left(f_{a p p}^{N}-f_{0}\right) \varphi d \theta d v+\iint\left(f-f_{a p p}^{N}\right) \varphi d \theta d v \\
& \geqslant \delta \iint f_{1} \varphi d \theta d v-\sum_{k=2}^{N} \delta^{k}\left\|f_{k}\right\|_{L^{1}}-\check{C}_{N}\left(\delta e^{t \operatorname{Re} \gamma}\right)^{N+1} \\
& \geqslant \delta \iint f_{1} \varphi d \theta d v-\sum_{k=2}^{N} C_{k}\left(\delta e^{t \operatorname{Re} \gamma}\right)^{k}-\check{C}_{N}\left(\delta e^{t \operatorname{Re} \gamma}\right)^{N+1} \\
& \geqslant \delta \iint f_{1} \varphi d \theta d v-2 K_{N}\left(\delta e^{t \operatorname{Re} \gamma}\right)^{2}-\check{C}_{N}\left(\delta e^{t \operatorname{Re} \gamma}\right)^{N+1} \\
& \geqslant \delta e^{t \operatorname{Re} \gamma}\left(\operatorname{Re}\left(e^{i t \operatorname{Im} \gamma} z_{g}\right)-\left\|\left(1-\chi_{\delta}\right) g\right\|_{L^{1}}-2 K_{N} \delta e^{t \operatorname{Re} \gamma}-\check{C}_{N}\left(\delta e^{t \operatorname{Re} \gamma}\right)^{N}\right)
\end{aligned}
$$

Assume for a while that

$$
\operatorname{Re}\left(e^{i t \operatorname{Im} \gamma} z_{g}\right) \geqslant \frac{\operatorname{Re} z_{g}}{2} .
$$

We have

$$
\left\|f-f_{0}\right\|_{L^{1}} \geqslant \frac{\delta e^{t \operatorname{Re} \gamma} \operatorname{Re} z_{g}}{2}\left(1-\frac{2\left\|\left(1-\chi_{\delta}\right) g\right\|_{L^{1}}}{\operatorname{Re} z_{g}}-\frac{4 K_{N} \delta e^{t \operatorname{Re} \gamma}}{\operatorname{Re} z_{g}}-\frac{2 \check{C}_{N}\left(\delta e^{t \operatorname{Re} \gamma}\right)^{N}}{\operatorname{Re} z_{g}}\right)
$$

Let $\delta_{0}>0$ be such that

$$
\frac{32 K_{N}}{\left(\operatorname{Re} z_{g}\right)^{2}} \delta_{0}+\frac{2 \breve{C}_{N} 8^{N}}{\left(\operatorname{Re} z_{g}\right)^{N+1}} \delta_{0}^{N} \leqslant \frac{1}{4} \quad \text { and } \quad \frac{8 \delta_{0}}{\operatorname{Re} z_{g}} \leqslant \min \left(\frac{1}{2}, \frac{1}{2 K_{N}}\right)
$$

(note that $N \geqslant 1$ ) and consider times $t$ such that

$$
\frac{4 \delta_{0}}{\operatorname{Re} z_{g}} \leqslant \delta e^{t \operatorname{Re} \gamma} \leqslant \frac{8 \delta_{0}}{\operatorname{Re} z_{g}} .
$$


Owing to (3.19), we also choose $\delta$ small enough such that

$$
\frac{2\left\|\left(1-\chi_{\delta}\right) g\right\|_{L^{1}}}{\operatorname{Re} z_{g}} \leqslant \frac{1}{4}
$$

We conclude from these inequalities that

$$
\left\|f-f_{0}\right\|_{L^{1}} \geqslant \delta_{0}
$$

and that (3.16) is satisfied.

To end the proof, it remains to fix the time $t_{\delta}$ and to choose the truncation function $\chi_{\delta}$. Let us show that, for $\delta$ small enough, there exists a time $t_{\delta}$ satisfying both (3.20) and (3.21). If $\operatorname{Im} \gamma=0$, then (3.20) is clearly satisfied since $\operatorname{Re} z_{g}>0$ : a suitable $t_{\delta}$ is then

$$
t_{\delta}=\frac{1}{\operatorname{Re} \gamma} \log \left(\frac{6 \delta_{0}}{\delta \operatorname{Re} z_{g}}\right) .
$$

Assume now that $\operatorname{Im} \gamma \neq 0$. For $\delta$ small enough, the size of the interval of times $t$ satisfying (3.21) becomes larger than $\frac{2 \pi}{|\operatorname{Im} \gamma|}$. This means that it is possible to find a time $t_{\delta}$ in this interval satisfying (3.20).

Step 5. Choice of $\chi_{\delta}$. For all $\delta>0$, we have to fix the function $\chi_{\delta} \in \mathcal{C}^{\infty}(\mathbb{R})$ such that (3.15), (3.17), (3.19) are satisfied and such that $f_{0}+\delta f_{1}(0) \geqslant 0$. First of all, proceeding as in the proof of Lemma 2.1, we obtain that $g$ takes the form

$$
g(\theta, v)=-m F^{\prime}\left(e_{0}\right) \cos \theta-m F^{\prime}\left(e_{0}\right) \int_{-\infty}^{0} \gamma e^{\gamma s} \cos \Theta(s) d s
$$

with $m=\int_{0}^{2 \pi} \rho_{f}(\theta) \cos \theta d \theta$. Hence,

$$
|g(\theta, v)| \leqslant|m|\left(1+\frac{|\gamma|}{\operatorname{Re} \gamma}\right)\left|F^{\prime}\left(e_{0}\right)\right| .
$$

The assumptions on $F$ and $F^{\prime}$ in Theorem 1.2 imply that

$$
\forall e<e_{*}, \quad\left|F^{\prime}(e)\right| \leqslant C\left(e_{*}-e\right)^{-\alpha} F(e)
$$

with $\alpha \geqslant 1$. Since $F(e)>0$ for $e<e_{*}$, the local assumption becomes global. Let $\chi$ be a $\mathcal{C}^{\infty}$ function such that $0 \leqslant \chi \leqslant 1$ and

$$
\begin{cases}\chi(t)=0 & \text { for } t \leqslant 0 \\ \chi(t) \leqslant 2 t^{\alpha} & \text { for } t \geqslant 0 \\ \chi(t)=1 & \text { for } t \geqslant 1\end{cases}
$$

and let

$$
\chi_{\delta}(e)=\chi\left(\frac{e_{*}-e}{\delta^{1 /(2 \alpha)}}\right) .
$$


From

$$
\left\|\left(1-\chi_{\delta}\right) g\right\|_{L^{1}} \leqslant\left\|g \mathbb{1}_{e_{*}-\delta^{1 /(2 \alpha)}<e_{0}(\theta, v)<e_{*}}\right\|_{L^{1}}
$$

and dominated convergence, we clearly have (3.19). By (3.23) and (3.24), we have, for all $(\theta, v) \in \mathbb{T} \times \mathbb{R}$,

$$
\delta\left|\operatorname{Re} g(\theta, v) \chi_{\delta}\left(e_{0}(\theta, v)\right)\right| \leqslant \delta C\left|e_{*}-e_{0}\right|^{-\alpha} F\left(e_{0}\right) \frac{\left|e_{*}-e_{0}\right|^{\alpha}}{\delta^{1 / 2}}=C \delta^{1 / 2} f_{0}(\theta, v),
$$

so for $\delta$ small enough, we have $f_{0}+\delta f_{1}(0) \geqslant 0$.

By differentiating (3.22) and using (3.7), we get

$$
\forall j+\ell \leqslant N, \quad\left|\partial_{\theta}^{j} \partial_{v}^{\ell} g(\theta, v)\right| \leqslant C \max _{k \leqslant N+1} F^{(k)}\left(e_{0}\right) \leqslant C\left|e_{*}-e_{0}\right|^{N-1},
$$

where we used Taylor formulas and the fact that $F \in \mathcal{C}^{\infty}$ with $F(e)=0$ for $e \geqslant e_{*}$. Besides, from (3.25), we obtain (if $\delta \leqslant 1$ )

$$
\forall 1 \leqslant j+\ell \leqslant N, \quad\left|\partial_{\theta}^{j} \partial_{v}^{\ell} \chi_{\delta}\left(e_{0}(\theta, v)\right)\right| \leqslant C \delta^{-N /(2 \alpha)} \mathbb{1}_{e_{*}-\delta^{1 /(2 \alpha)}<e_{0}(\theta, v)<e_{*}} .
$$

Therefore

$$
\begin{gathered}
\left\|g \chi_{\delta}\right\|_{W^{N, 1}} \leqslant\|g\|_{L^{1}}+C \delta^{-N /(2 \alpha)} \int_{0}^{2 \pi} \int_{\mathbb{R}}\left|e_{*}-e_{0}(\theta, v)\right|^{N-1} \mathbb{1}_{e_{*}-\delta^{1 /(2 \alpha)}<e_{0}(\theta, v)<e_{*}} d \theta d v \\
\leqslant\|g\|_{L^{1}}+C \delta^{-N /(2 \alpha)} \int_{e_{*}-\delta^{1 /(2 \alpha)}}^{e_{*}}\left(e_{*}-e\right)^{N-1}\left(4 \int_{0}^{\theta_{m_{0}}} \frac{d \theta}{\sqrt{2\left(e+m_{0} \cos \theta\right)}}\right) d e \\
=\|g\|_{L^{1}}+C \delta^{-N /(2 \alpha)} \int_{e_{*}-\delta^{1 /(2 \alpha)}}^{e_{*}}\left(e_{*}-e\right)^{N-1} T_{e} d e,
\end{gathered}
$$

where $\theta_{m_{0}}=\arccos \left(-\frac{e}{m_{0}}\right)$ and $T_{e}$ is given by (2.9). Now we recall that for $e \leqslant e_{*}$ we have $T_{e} \leqslant T_{e_{*}}$. This yields

$$
\left\|g \chi_{\delta}\right\|_{W^{N, 1}} \leqslant\|g\|_{L^{1}}+C T_{e_{*}} \delta^{-N /(2 \alpha)} \int_{e_{*}-\delta^{1 /(2 \alpha)}}^{e_{*}}\left(e_{*}-e\right)^{N-1} d e=\|g\|_{L^{1}}+\frac{C T_{e_{*}}}{N} .
$$

We have proved (3.15).

By (3.9), we have

$$
\begin{aligned}
\left\|\widetilde{R}_{\delta}\right\|_{L^{1}} & \leqslant C\left(\left\|\left(1-\chi_{\delta}\right) g\right\|_{L^{1}}+\left\|\left(1-\chi_{\delta}\right) \partial_{v} f_{0}\right\|_{L^{1}}\right) \\
& \leqslant C\left(\left\|g \mathbb{1}_{e_{*}-\delta^{1 /(2 \alpha)}<e_{0}(\theta, v)<e_{*}}\right\|_{L^{1}}+\left\|\partial_{v} f_{0} \mathbb{1}_{e_{*}-\delta^{1 /(2 \alpha)}<e_{0}(\theta, v)<e_{*}}\right\|_{L^{1}}\right),
\end{aligned}
$$

so by dominated convergence,

$$
\lim _{\delta \rightarrow 0}\left\|\widetilde{R}_{\delta}\right\|_{L^{1}}=0 .
$$

We now choose $\delta$ small enough such that

$$
\left\|R_{\delta}\right\|_{L^{1}} \leqslant\left(\frac{4 \delta_{0}}{\operatorname{Re} z_{g}}\right)^{N} .
$$

From (3.21), we obtain (3.17), which ends the proof of Theorem 1.2 


\section{A Appendix. Existence of unstable steady states}

In this section, we prove that the set of steady states satisfying the assumptions of Theorems 1.1 and 1.2 is not empty. More precisely, we prove the following

Lemma A.1. Let $m>0$. There exist $m>0, e_{*}<m$ and there exists a nonincreasing function $F, \mathcal{C}^{\infty}$ on $\mathbb{R}$, such that $F(e)>0$ for $e<e_{*}, F(e)=0$ for $e \geqslant e_{*}$ and $\left|F^{\prime}(e)\right| \leqslant C\left|e_{*}-e\right|^{-\alpha} F(e)$ in the neighborhood of $e_{*}$, for some $\alpha \geqslant 1$, and such that the function $f(\theta, v)=F\left(\frac{v^{2}}{2}-m \cos \theta\right)$ is a steady state solution to the HMF model (1.1) and such that $\kappa(m, F)>1$, where $\kappa(m, F)$ is given by

$\kappa(m, F)=\int_{0}^{2 \pi} \int_{-\infty}^{+\infty}\left|F^{\prime}(e(\theta, v))\right|\left(\frac{\int_{\mathcal{D}_{e(\theta, v)}}\left(\cos \theta-\cos \theta^{\prime}\right)\left(e(\theta, v)+m \cos \theta^{\prime}\right)^{-1 / 2} d \theta^{\prime}}{\int_{\mathcal{D}_{e(\theta, v)}}\left(e(\theta, v)+m \cos \theta^{\prime}\right)^{-1 / 2} d \theta^{\prime}} d \theta d v\right.$

with

$$
e(\theta, v)=\frac{v^{2}}{2}-m \cos \theta, \quad \mathcal{D}_{e}=\left\{\theta^{\prime} \in \mathbb{T}: m \cos \theta^{\prime}>-e\right\} .
$$

Proof. Let $m>0$ and $F$ a nonincreasing $\mathcal{C}^{\infty}$ function on $\mathbb{R}$ supported in $(-\infty, m)$, which is not identically zero on $(-m, m)$. We first observe that $f(\theta, v)=F\left(\frac{v^{2}}{2}-m \cos \theta\right)$ is a steady state solution to the HMF model (1.1) if and only if $m$ and $F$ satisfy $\gamma(m, F)=m$ with

$$
\gamma(m, F):=\int_{0}^{2 \pi} \int_{\mathbb{R}} F\left(\frac{v^{2}}{2}-m \cos \theta\right) \cos \theta d \theta d v>0 .
$$

By using the linearity of $\gamma$ in $F$ we deduce that $\frac{m}{\gamma(m, F)} F\left(\frac{v^{2}}{2}-m \cos \theta\right)$ is a steady state.

We proceed by a contradiction argument. Assume that

$$
\kappa\left(m, \frac{m}{\gamma(m, F)} F\right) \leqslant 1
$$

for all $m>0$ and all nonincreasing $\mathcal{C}^{\infty}$ function $F$ supported in $(-\infty, m)$ such that, denoting by $\left(-\infty, e_{*}\right]$ the support of $F$, we have $\left|F^{\prime}(e)\right| \leqslant C\left|e_{*}-e\right|^{-\alpha} F(e)$ in the neighborhood of $e_{*}$, for some $\alpha \geqslant 1$. This is equivalent to

$$
\kappa(m, F) \leqslant \frac{\gamma(m, F)}{m},
$$

or, after straightforward calculation and an integration by parts,

$$
-\iint F^{\prime}(e(\theta, v)) g_{m}(e(\theta, v)) d \theta d v \leqslant 0
$$

with

$$
g_{m}(e)=\left(\Pi_{m} \cos ^{2} \theta\right)(e)-\left(\left(\Pi_{m} \cos \theta\right)(e)\right)^{2}-\left(\Pi_{m} \sin ^{2} \theta\right)(e)
$$


and for all function $h(\theta)$,

$$
\left(\Pi_{m} h\right)(e)=\frac{\int_{\mathcal{D}_{e}}(e+m \cos \theta)^{-1 / 2} h(\theta) d \theta}{\int_{\mathcal{D}_{e}}(e+m \cos \theta)^{-1 / 2} d \theta} .
$$

Now, we choose the functions $F$ as follows. We first pick a nonincreasing $\mathcal{C}^{\infty}$ function $\Psi$ on $\mathbb{R}$ with support $\left(\infty, e_{\sharp}\right] \subset(-\infty, m)$, then we set $e_{*}=\frac{e_{\sharp}+m}{2}$ and define

$$
F_{\varepsilon}(e)=\Psi(e)+\varepsilon \exp \left(-\left(e_{*}-e\right)^{-1}\right), \quad \text { for } e<e_{*},
$$

the parameter $\varepsilon>0$ being arbitrary. Since $F_{\varepsilon}$ satisfies the assumptions, it satisfies (A.1). Then, letting $\varepsilon \rightarrow 0$, we get

$$
-\iint \Psi^{\prime}(e(\theta, v)) g_{m}(e(\theta, v)) d \theta d v \leqslant 0
$$

The function $\Psi$ being arbitrary, this is equivalent to

$$
g_{m}(e) \leqslant 0, \quad \forall m>0, \quad \forall e \in(-m, m),
$$

or,

$$
g_{1}(e) \leqslant 0, \quad \forall e \in(-1,1) .
$$

Let us now prove that the function $g_{1}(e)$ is in fact positive in the neighborhood of $e=1$, which contradicts (A.2).

Indeed, we introduce

$$
\alpha(e)=\int_{\mathcal{D}_{e}}(e+\cos \theta)^{-1 / 2} d \theta, \quad \beta(e)=\int_{\mathcal{D}_{e}}(e+\cos \theta)^{-1 / 2} \sin ^{2} \theta d \theta .
$$

We have

$$
\begin{aligned}
\alpha(e) g_{1}(e) & =\alpha(e)-2 \beta(e)-\frac{1}{\alpha(e)}\left(\int_{\mathcal{D}_{e}}(e+\cos \theta)^{1 / 2} d \theta-e \alpha(e)\right)^{2} \\
& =\left(1-e^{2}\right) \alpha(e)-2 \beta(e)+2 e \int_{\mathcal{D}_{e}}(e+\cos \theta)^{1 / 2} d \theta-\frac{1}{\alpha(e)}\left(\int_{\mathcal{D}_{e}}(e+\cos \theta)^{1 / 2} d \theta\right)^{2} .
\end{aligned}
$$

From [22], we have

$$
\alpha(e) \sim-\sqrt{2} \log (1-e) \quad \text { as } e \rightarrow 1^{-},
$$

and direct calculations yield

$$
\int_{0}^{2 \pi}(1+\cos \theta)^{1 / 2} d \theta=4 \sqrt{2}, \quad \beta(1)=\frac{8 \sqrt{2}}{3} .
$$

This means that

$$
\begin{aligned}
\alpha(e) g_{1}(e) & \rightarrow \frac{8 \sqrt{2}}{3}>0 \quad \text { as } e \rightarrow 1^{-}, \\
g_{1}(e) & \sim \frac{8 \sqrt{2}}{\alpha(e)} \text { as } e \rightarrow 1^{-} .
\end{aligned}
$$

This proves the claim. 


\section{Acknowledgments}

The authors wish to thank D. Han-Kwan for helpful discussions. A. M. Luz acknowledges support by the Brazilian National Council for Scientific and Technological Development (CNPq) under the program "Science without Borders" 249279/2013-4. M. Lemou and F. Méhats acknowledge supports from the ANR project MOONRISE ANR-14-CE23-000701, from the ENS Rennes project MUNIQ and from the INRIA project ANTIPODE.

\section{References}

[1] M. Antoni, S. Ruffo, Clustering and relaxation in Hamiltonian long-range dynamics, Phys. Rev. E, 52 (1995), 2361.

[2] A. Antoniazzi, D. Fanelli, S. Ruffo, Y. Y. Yamaguchi, Nonequilibrium tricritical point in a system with long-range interactions, Phys. Rev. Lett. 99 (2007), 040601.

[3] J. Barré, F. Bouchet, T. Dauxois, S. Ruffo, Y. Y. Yamaguchi, The Vlasov equation and the Hamiltonian mean-field model, Physica A 365 (2006), 177.

[4] J. Barré, A. Olivetti, Y. Y. Yamaguchi, Dynamics of perturbations around inhomogeneous backgrounds in the HMF model, J. Stat. Mech. (2010), 08002.

[5] J. Barré, A. Olivetti, Y. Y. Yamaguchi, Algebraic damping in the one-dimensional Vlasov equation, J. Phys. A: Math. Gen. 44 (2011), 405502.

[6] J. Barré, Y. Y. Yamaguchi, Small traveling clusters in attractive and repulsive Hamiltonian mean-field models, Phys. Rev. E 79 (2009), 036208.

[7] J. Barré, Y. Y. Yamaguchi, On the neighborhood of an inhomogeneous stable stationary solution of the Vlasov equation - Case of an attractive cosine potential, J. Math. Phys. 56 (2015), 081502.

[8] E. Caglioti, F. Rousset, Long time estimates in the mean field limit, Arch. Ration. Mech. Anal. 190 (2008), no. 3, 517-547.

[9] E. Caglioti, F. Rousset, Quasi-stationary states for particle systems in the mean-field limit, J. Stat. Phys. 129 (2007), no. 2, 241-263.

[10] A. Campa, P.-H. Chavanis, Inhomogeneous Tsallis distributions in the HMF model, J. Stat. Mech. (2010), 06001.

[11] P.-H. Chavanis, Lynden-Bell and Tsallis distributions for the HMF model, Eur. Phys. J. B 53 (2006), 487.

[12] P.-H. Chavanis, J. Vatteville, F. Bouchet. Dynamics and thermodynamics of a simple model similar to self-gravitating systems : the HMF model, Eur. Phys. J. B, 46 (2005), 61. 
[13] E. Faou, F. Rousset, Landau damping in Sobolev spaces for the Vlasov-HMF model, Arch. Ration. Mech. Anal. 219 (2016), no. 2, 887-902.

[14] E. Grenier, On the nonlinear instability of Euler and Prandtl equations, Comm. Pure Appl. Math. 53 (2000), no. 9, 1067-1091.

[15] Y. Guo, Z. Lin, Unstable and stable Galaxy models, Comm. Math. Phys. 279 (2008), 789-813.

[16] Y. Guo, W. Strauss, Nonlinear instability of double-humped equilibria, Ann. Inst. H. Poincaré Anal. Non Linéaire 12 (1995), 339-352.

[17] D. Han-Kwan, M. Hauray, Stability issues in the quasineutral limit of the onedimensional Vlasov-Poisson equation, Comm. Math. Phys. 334 (2015), no. 2, 11011152 .

[18] D. Han-Kwan, T. Nguyen, Instabilities in the mean field limit, J. Stat. Phys. 162 (2016), no. 6, 1639-1653.

[19] Z. Lin, Instability of periodic BGK waves, Math. Res. Letts. 8 (2001), 521-534.

[20] M. Lemou, F. Méhats, P. Raphaël, Structure of the linearized gravitational VlasovPoisson system close to a polytropic ground state, SIAM J. Math. Anal. 39 (2008), no. $6,1711-1739$.

[21] M. Lemou, F. Méhats, P. Raphaël, Orbital stability of spherical galactic models, Inventiones Math. 187 (2012), 145-194.

[22] M. Lemou, A. M. Luz, F. Méhats, Nonlinear stability criteria for the HMF Model, Arch. Rational Mech. Anal. 224 (2017), no. 2, 353-380.

[23] S. Ogawa, Spectral and formal stability criteria of spatially inhomogeneous solutions to the Vlasov equation for the Hamiltonian mean-field model, Phys. Rev. E 87 (2013), 062107.

[24] S. Ogawa and Y. Y. Yamaguchi, Precise determination of the nonequilibrium tricritical point based on Lynden-Bell theory in the Hamiltonian mean-field model, Phys. Rev. E, 84 (2011), 061140.

[25] Y. Shizuta, On the classical solutions of the Boltzmann equation, Comm. Pure Appl. Math. 36 (1983), no. 6, 705-754.

[26] F. Staniscia, P. H. Chavanis, G. De Ninno, Out-of-equilibrium phase transitions in the HMF model : a closer look, Phys. Rev. E. 83 (2011), 051111.

[27] Y. Y. Yamaguchi, Construction of traveling clusters in the Hamiltonian mean-field model by nonequilibrium statistical mechanics and Bernstein-Greene-Kruskal waves, Phys. Rev. E 84 (2011), 016211. 
[28] Y. Y. Yamaguchi, J. Barré, F. Bouchet, T. Dauxois, S. Ruffo, Stability criteria of the Vlasov equation and quasi-stationary states of the HMF model, Physica A 337 (2004), 36 . 